\title{
Arznei- und Heilpflanzen: Ein abgestuftes und globales Arzneimittelangebot
}

Es gibt weltweit kaum eine Heilpflanze oder irgendeine andere Pflanze, die gerade als Heilmittel propagiert wird, deren einfache Zubereitungen nicht für Patienten oder Konsumenten erhältlich wären. Die Bezugsquellen sind vielfältig (z.B. offizielle Importe, zumeist jedoch Grau- und Schwarzimporte, Bezugsmöglichkeiten über Internetadressen). Handelt es sich um aktuell «aussereuropäische» Pflanzen, so besitzen die daraus hergestellten Produkte zumeist keinen Arzneimittelstatus bzw. werden in vielen Ländern der Ersten Welt nicht als Arzneimittel akzeptiert, selbst wenn sie in den Ursprungsländern die Anerkennung als Arzneimittel erworben haben $[1,2]$. Entsprechend dieser Situation sind Qualität und Sicherheit der bezogenen Mittel ausserordentlich unterschiedlich, immer wieder wechselnd und v.a. kaum voraussagbar. Auch heillose Fälschungen erreichen leicht Patient und Konsument. Die derzeitige Situation bedeutet einen erheblichen Verzicht auf prinzipiell mögliche Qualität und Sicherheit.

Eine Zulassung als Arzneimittel könnte zumindest für kranke Menschen vielen dieser Risiken vorbeugen. Die Forderung nach einem Arzneimittelstatus bleibt aber illusorisch, wenn es in der Realität überhaupt nicht bzw. nicht mit den verfügbaren Mitteln möglich ist, diesen Status mit den daraus resultierenden Verpflichtungen zu erlangen. Die stetig anschwellenden Forderungen und ökonomischen Voraussetzungen für eine Registrierung neuer Arzneimittel sind allenfalls für eine kleine Zahl zumeist transnationaler Konzerne erfüllbar. Damit ist es ihnen vielfach überlassen, durch die Entwicklung bzw. Nichtentwicklung von Arzneimitteln, indirekt materielle und zentrale Inhalte der Medizin und damit von Gesundheit und Krankheit zu gestalten. Es besteht die sehr konkrete Gefahr der Monopolisierung der Arzneimittelentwicklung und der damit zusammenhängenden medizinischen Denkstile und Theorien.

Auch für die Phytotherapie der Länder der Ersten Welt scheint es derzeit allenfalls selten möglich zu sein, Innovatio- nen zur Zulassung zu bringen oder in Zulassungshinsicht das vielfältige Potential selbst von bekannten Arzneipflanzen (biogene Vielstoffgemische [3, 4] als potentielle biologische sowie «kulturelle» Rohstoffe für Arzneimittel mit unterschiedlichen Wirkungsspektren) zu entwickeln. Arznei- und Heilpflanzen besitzen nicht nur wie andere Arzneimittel eine relativ kurze, wenngleich mittlerweile auch intensive Geschichte aus dem Labor (naturwissenschaftlicher Forschungsstand). Sie transportieren zudem mannigfaltige Erfahrungen (Überlieferungsbestände), Betrachtungsweisen von Gesundheit und vor allem therapeutische Anregungen aus Jahrhunderten der Anwendung sowie regionalen Medizinsystemen [1]. So ist z.B. Johanniskraut im Vergleich zu seiner Anwendungsgeschichte seit relativ kurzer Zeit als ein Antidepressivum etabliert und zugelassen. Wesentlich länger wurde es umfangreich als Nerven-, Rekonvaleszenz- und Konstitutionsmittel gebraucht [5]. Der damit verbundene, teils vitalistische, teils funktionelle Konstitutionsbegriff (Konstitution als Mischung aus abwehrender Energie und rezeptiver Kraft) und die therapeutische Intention (Versuch der Mobilisierung körperlicher, aber auch psychischer Energien) finden keine konventionelle krankheitsspezifische Entsprechung. Trotzdem sind diese Gesichtspunkte, die für eine Vielzahl pflanzlicher Heilmittel zutreffen, für moderne Behandlungen fruchtbar. Die Entwicklung zulassungsreifer Arzneimittel gehorcht keineswegs nur einem therapeutischen Imperativ, sondern mindestens genauso intensiv wirtschaftlichen Erwartungen und politisch beförderten regulatorischen Vorgaben.

Wesentlicher schwieriger ist noch die Situation für zum Teil bereits seit Jahrzehnten global - und damit auch z.B. in Europa - eingenommene Produkte aus Arznei- und Heilpflanzen anderer, nichtwestlicher Medizinsysteme (z.B. in Form irgendwelcher «Ergänzungsmittel» $[6,7])$. Die gerne und scheinbar kritisch, oft gebetsmühlenhaft wiederholten Forderungen, solche pflanzlichen Mittel sollten materiell und ideologisch aus-

\begin{tabular}{ll}
\hline KARGER & @ 2004 S. Karger GmbH, Freiburg \\
$\begin{array}{l}\text { Fax +49 76145207 14 } \\
\begin{array}{l}\text { E-mail Information@Karger.de } \\
\text { www.karger.com }\end{array}\end{array}$ & $\begin{array}{l}\text { Accessible online at: } \\
\text { www.karger.com/fkm }\end{array}$
\end{tabular}

Prof. Dr. med. Reinhard Saller 
schliesslich genauso behandelt werden wie krankheitsspezifisch neu entwickelte, pathophysiologisch einfacher handhabbare Monosubstanzen bzw. einfache «Kunstmischungen», bedeuten zumeist das regulatorische «Aus». Die wirtschaftlichen Anforderungen für eine Zulassung können dann den «Stop» zementieren. Alle Erfahrungen zeigen, dass Mittel aus solchen Pflanzen trotzdem weiterhin eingenommen werden, allerdings ohne den Schutz der regulierten Qualität eines Arzneimittels.

Das Interesse grosser Bereiche der konventionellen Medizin an solchen Pflanzen konzentriert sich auf sogenannte Bioprospektionsprojekte, d.h. auf die gezielte multidisziplinäre Erkundung und Sammlung des pflanzlichen Materials sowie dessen Aufbereitung. Die gesammelten Extrakte werden mittels technischer Verfahren, zum Teil mit Hilfe des genetischen Screenings, auf ihre Inhaltsstoffe und deren Anwendungsmöglichkeiten hin für eine zukünftige gewerbliche Nutzung untersucht. Dies entspricht zu einem grossen Teil einer klassischen Naturstoffforschung bzw. der Suche nach Heilpflanzen, jedoch entsprechend dem Bedarf der Ersten Welt, zur Entwicklung gewinnträchtiger und patentierbarer Arzneimittel [8, 9]. Durch Bioprospektionsprojekte lässt sich die Ausbeute von Arzneimittelprojekten erheblich erhöhen, z.B. 1 von 10 Projekten anstelle einer konventionellen Ausbeute von 1 auf 50 Projekte. Die mögliche wirtschaftliche Bedeutung zeigt sich etwa am Beispiel eines «Schlankmachers aus Afrika» (Wirkstoffe aus dem von den San traditionell genutzten HoodiaKaktus). Der potentielle Marktwert eines Arzneimittels auf dieser Basis wird je nach Rezeptpflicht bzw. Freiverkäuflichkeit auf 4-30 Milliarden \$ geschätzt. Es ist nachvollziehbar, dass ein Teil solcher «Privatisierungen» traditionellen Wissens von verschiedenen Betroffenen eher als eine moderne Form von Biopiraterie gesehen wird. Mit einer Erweiterung genuiner phytotherapeutischer Möglichkeiten hat eine solche Bioprospektion nichts zu tun

Für eine ganzheitliche, d.h. komplexe, naturheilkundlich orientierte Erweiterung der Phytotherapie, mit Erfahrungen und Produkten anderer Kulturen, spielen auch Gesichtspunkte der Nachhaltigkeit eine Rolle, die als Fairer Handel (fair trade) $[10,11]$ und ökologischer Landbau [12] von Nahrungspflanzen bekannt sind, und im Rahmen von Pilotprojekten mit Arznei- und Heilpflanzen aus der Dritten Welt Anwendung finden. Dies bedeutet z.B. auch eine differenzierte Betrachtung und Abwägung der Produktionsbedingungen in den Ländern der Dritten Welt. Die undifferenzierten kategorischen Forderungen nach einer sozusagen klinisch-sterilen Herstellung ausschliesslich im Sinne durchrationalisierter westlicher Labors bedeuten für einen Grossteil der bislang Beschäftigten Arbeitslosigkeit mit all ihren Konsequenzen. Während die bisherigen relativ starren Zulassungsvorschriften mit ihren auf biomedizinische Produkte gezielten quantitativen Anforderungen in der Praxis kaum Erweiterungen der arzneilichen Phytotherapie und einen authentischen transkulturellen Austausch von Therapieansätzen erlaubten, könnten die verabschiedeten Richtlinien der EU über «traditional use» [13] und «well-established medicinal use» [14] der Phytotherapie neue und zukunftsträchtige Möglichkeiten erschliessen. Allerdings ist ein realitätsgerechter und dennoch phantasievoller Umgang nötig, um z.B. Patienten zu ermöglichen, gesichtete Heilmittel aus Pflanzen z.B. der ayurvedischen Medizin mit der Qualität und Sicherheit von Arzneimitteln einnehmen zu können. Dem jeweiligen Verwendungsgebiet angemessene Qualität und Sicherheit zu dokumentieren und zu gewährleisten ist eine selbstverständliche und v.a. erfüllbare Aufgabe eines Arzneimittelherstellers, ebenso die eindeutige Angabe der jeweiligen Empirie für die verschiedenen Anwendungsbereiche. Eine starre Fixierung der Zulassung an konventionelle Indikationen im Sinne biomedizinischer Nosologien dürfte viele der angesprochenen Probleme ungelöst lassen. Konventionelle kontrollierte klinische Studien für jedwedes traditionelle Arzneimittel mit vielfältigen Verwendungsmöglichkeiten als conditio sine qua non zu fordern ist wissenschaftlich und ökonomisch unsinnig. Geht man realitätsbezogen, vorurteilsfrei und therapeutisch aufgeschlossen mit diesen Möglichkeiten um, kann heutzutage die Anerkennung von Erfahrungen und Verwendungen einer Droge nicht ernsthaft geografisch auf Länder der Ersten Welt eingeschränkt werden.

Zweifellos ist jeder Versuch, Qualität und Sicherheit von Therapiemitteln $\mathrm{zu}$ erhöhen, besser als der jetzige Zustand, der entscheidungswillige und entscheidungsfähige Patienten den Grau- und Schwarzmärkten und qualitativ ungeprüften Produkten überlässt. Auch für Ärzte brächten solche Entwicklungen eindeutige Vorteile. Einerseits stünden qualitativ bessere Produkte zur Verfügung, und andererseits könnten sie das Spektrum ihrer phytotherapeutischen Therapiemöglichkeiten ohne Qualitätsunsicherheiten ausweiten. Für die Phytotherapie sind solche Aspekte von Weltoffenheit («Globalisierung») und therapeutischer Neugierde keineswegs neu. Die verschiedenen geografisch eingegrenzten Phytotherapierichtungen haben bereits vor Jahrhunderten Pflanzen und Behandlungsansätze ausgetauscht und sich gegenseitig befruchtet.

Die neuen Möglichkeiten für Phytotherapeutika bedeuten keineswegs einen Verzicht auf qualitativ angemessene und damit hoch stehende Forschung. Auch biomedizinisch ausgerichtete klinische Forschung, z.B. vergleichende kontrollierte Studien, bleibt, entsprechend den Richtlinien, für eine Reihe von Anwendungssituationen selbstverständlich notwendig. Es ist dringend zu wünschen, dass die offensichtlichen Möglichkeiten dieser Richtlinien sowohl von den Regierungen und Zulassungsbehörden wie auch von phytotherapeutisch orientierten Firmen und den Ärzten und Therapeuten angemessen, nachvollziehbar und phantasievoll aufgegriffen, umgesetzt und weiter entwickelt werden.

R. Saller, J. Melzer, Zürich 


\section{Literatur}

1 Shankar D: Conserving the medicinal plants of India: the need for a biocultural perspective. $\mathrm{J} \mathrm{Al}$ tern Complement Med 1996;2:349-358.

2 Normile D: Asian medicine. The new face of traditional Chinese medicine. Science 2003;299:188-190.

3 Saller R: Cannabis and Marihuana als Vielstoffgemische in der Phytotherapie. Forsch Komplementärmed 1999;6(suppl 3):3-6.

4 Meier B: Von der Arzneipflanze zum Phytotherapeutikum. Ther Umsch 2002;59:275-282.

5 Saller R, Melzer J, Reichling J: Johanniskraut (Hypericum perforatum): Ein plurivalenter Rohstoff für traditionelle und moderne Therapien. Forsch Komplementärmed Klass Naturheilkd 2003; 10(suppl 1):33-40.

6 Zeisel SH: Regulation of 'nutraceuticals'. Science 1999;285:1853-1855.
Colic M, Pavelic K: Molecular, cellular and medical aspects of the action of nutraceuticals and small molecules therapeutics: from chemoprevention to new drug development. Drugs Exp Clin Res 2002; 28:169-175.

8 Myles DC: Novel biologically active natural and unnatural products. Curr Opin Biotechnol 2003; 14:627-633.

9 Jagtenberg T, Evans S: Global herbal medicine: a critique. J Altern Complement Med 2003;9: 321-329.

10 Chen J: Challenges to developing countries after joining WTO: risk assessment of chemicals in food. Toxicology 2004;198:3-7.

11 Thiermann AB: Protecting health, facilitating trade, or both? Ann N Y Acad Sci 2000;916:24-30.
2 Mäder P, Fliessbach A, Dubois D, Gunst L, Fried P, Niggli U: Soil fertility and biodiversity in organic farming. Science 2002;296:1694-1697.

13 Schauss AG: A review of the European food supplements and traditional herbal medicines directives. HerbalGram 2004;63:64-69.

14 EMEA (homepage on the internet), London, 1999 (cited 13.09.2004). Herbal Medicinal Products Working Party: Proposal for a note for guidance on non-clinical testing of herbal drug preparations with long-term marketing experience - guidance to facilitate mutual recognition and use of bibliographic data. Available from: http://www.emea.eu.int/ pdfs/human/hmpwp/001199en.pdf. 